\title{
Hybrid Mass Spectrometry Methods Reveal Lot-to-Lot Differences and Delineate the Effects of Glycosylation on the Structure of Herceptin ${ }^{\circledR}$
}

\author{
Rosie Upton ${ }^{1}$, Lukasz G. Migas ${ }^{1}$, Kamila J. Pacholarz ${ }^{1}$, Richard G. Beniston ${ }^{2}$, David Firth ${ }^{2}$, Sian \\ Estdale $^{2}$ and Perdita E. Barran ${ }^{1}$ \\ ${ }^{1}$ Manchester Institute of Biotechnology, Michael Barber Centre for Collaborative Mass Spectrometry, \\ University of Manchester, 131 Princess Street, Manchester, M1 7DN, United Kingdom \\ ${ }^{2}$ Covance Laboratories Ltd., Otley Road, Harrogate, HG3 1PY, United Kingdom
}

\begin{abstract}
To consider the measurable variations in biopharmaceuticals we use mass spectrometry and systematically evaluate three lots of Herceptin ${ }^{\circledR}$, two mAb standards and an intact Fc-hinge fragment. Each $\mathrm{mAb}$ is examined in three states; glycan intact, truncated (following endoS2 treatment) and fully deglycosylated. Despite equivalence at the protein level, each lot of Herceptin ${ }^{\circledast}$ gives a distinctive signature in three different mass spectrometry analyses. Ion mobility mass spectrometry (IM-MS) shows that in the API, the attached N-glycans reduce the conformational spread of each mAb by 10.5 $-25 \%$. Hydrogen/deuterium exchange mass spectrometry (HDX-MS) data supports this, with lower global deuterium uptake in solution when comparing intact to the fully deglycosylated protein. HDXMS and activated IM-MS map the influence of glycans on the mAb and reveal allosteric effects which extend far beyond the Fc domains into the Fab region. Taken together these findings, and the supplied interactive data sets could be used to provide acceptance criteria with application for MS based characterisation of biosimilars and novel therapeutic mAbs.
\end{abstract}

\section{Introduction}

As many as $70 \mathrm{mAb}$ products are predicted to be on the market by $2020 .{ }^{1}$ To date the majority are produced through heterogeneous mammalian cell lines ${ }^{2}$ which are responsible for transcribing post-translational modifications (PTMs) such as glycosylation. Subtle differences in the cell cultures and manufacturing conditions including $\mathrm{pH}$ and temperature can lead to variations in the glycan profiles and structure of the mAbs. ${ }^{3}$ This process-related variability can therefore lead to changes in the biological activity and pharmacokinetics.

As mAb products come off patent, the opportunity to develop biosimilar candidates arises however, because mAb biologics are large ( $\sim 150 \mathrm{kDa})$, flexible molecules that can exhibit intrinsic heterogeneity through PTMs, truncations, as well as global features such as fold and aggregation propensity ${ }^{4}$, the approval pathway for any new mAb or biosimilar is fraught with difficulty. This could 
be in part allayed with the development of robust analytical methods. Although guidelines are in place from the European Medicines Agency $(\mathrm{EMA})^{5,6}$, the United States Food and Drug Administration $(\mathrm{FDA})^{7,8}$ and the World Health Organisation $(\mathrm{WHO})^{9}$, equivalence acceptance criteria currently need to be considered on a case-by-case basis for each mAb. ${ }^{10}$ With this in mind, thorough characterisation of any innovator therapeutic is required to understand the effects of primary and higher order structure, PTMs and biological activity upon function, efficacy and safety. One critical assessment to make, with direct relevance to the development and licencing of new biopharmaceuticals as well as to biosimilar production, is a comparison between lots of a given mAb product.

N-linked glycosylation of the conserved Fc asparagine residue ("Asn-297") in mAbtherapeutics plays a key role in the immunological activity and structural conformation of the IgG1 protein. ${ }^{11-13}$ Furthermore, since we have demonstrated lot-to-lot variability in the glycosylation profile of Herceptin ${ }^{\circledR}$, it follows that measuring how glycosylation impacts the overall mAb conformation is of prime importance. Mass spectrometry based techniques have been widely used for the characterisation of antibodies ${ }^{14-16}$, quantification of glycosylation profiles ${ }^{17-19}$ and to study conformational changes that occur upon deglycosylation ${ }^{20,21}$. Hydrogen-deuterium exchange mass spectrometry (HDX-MS) and ion mobility mass spectrometry (IM-MS) have provided new insights to mAb structure, providing rapid analysis from only a few micrograms of sample, and the former enabling structural differences to be mapped to regions of the protein fold. ${ }^{21-24}$

We have investigated the hypotheses presented by Berkowitz et al. that MS is a 'valuable tool' for probing the three-dimensional higher order structure both for lot-to-lot comparisons and for studying the effects of PTMs upon conformation. ${ }^{13}$ We employ three mass spectrometry methods to compare the global conformation and dynamics for Herceptin ${ }^{\circledast}$, in three separately manufactured lots and for each at differing levels of Fc-domain N-linked glycosylation (Figure 1). We and others have previously demonstrated the use of an endoglycosidase, endoS2, to dissect between the two N-acetyl glucosamine residues of the chitobiose core, leaving only a single GlcNAc or [GIcNAc + Fucose] stub to aid afucosylation quantification ${ }^{18}$ and high-mannose quantification. ${ }^{25}$ By using endoS2 alongside PNGase $\mathrm{F}$ we can investigate the conformational changes and dynamics of IgG1 mAbs with three differing levels of glycosylation; fully intact, 'truncated' (endoS2 treated) and fully deglycosylated (PNGase F treated). We have used HDX-MS to study different lots of Herceptin ${ }^{\circledR}$, along with a mAb supplied by Waters as a mass check standard and an intact IgG1 Fc-hinge fragment. We find that upon glycan removal and 'truncation' there is an increase in deuterium uptake, indicative of an increased number of solvent accessible exchangeable amide hydrogens. For IM-MS studies, both collision cross section distributions (CCSD) and collision activated IM-MS heat maps are presented for different lots of Herceptin ${ }^{\circledR}$, the Fc-hinge fragment and also the NIST mAb reference material. ${ }^{26}$ The NIST mAb is a 
useful standard for the evaluation of lot-to-lot comparisons as it has been fully homogenised across multiple lots prior to its release. ${ }^{27}$ The glycosylation profiles and therefore the conformational properties of the reference material is predicted to be typically more varied than individual lots.

(c)

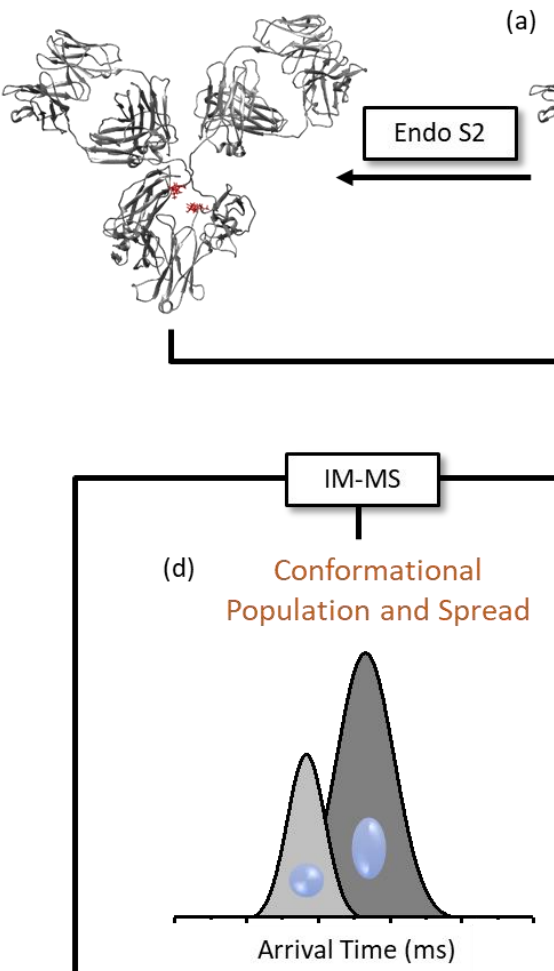

(e) Conformational Stability

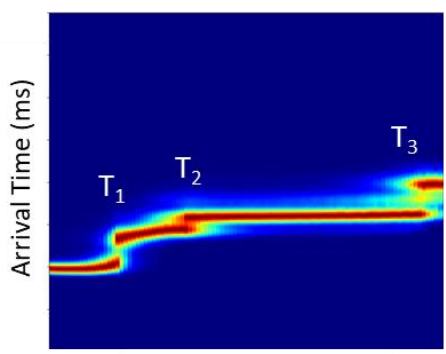

Collision Voltage (V)

(a)
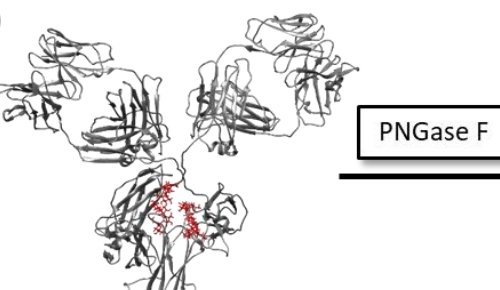

(b)

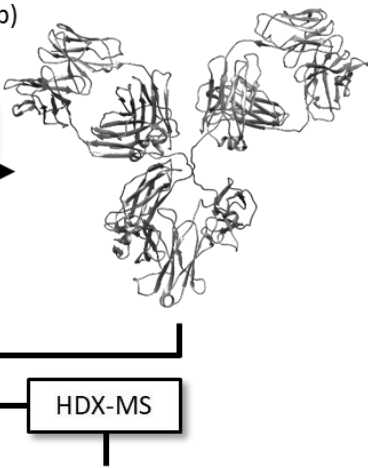

Native MS

(f) Localised Dynamics and Allosteric Effects

$\mathrm{D}_{2} \mathrm{O}$ labelled
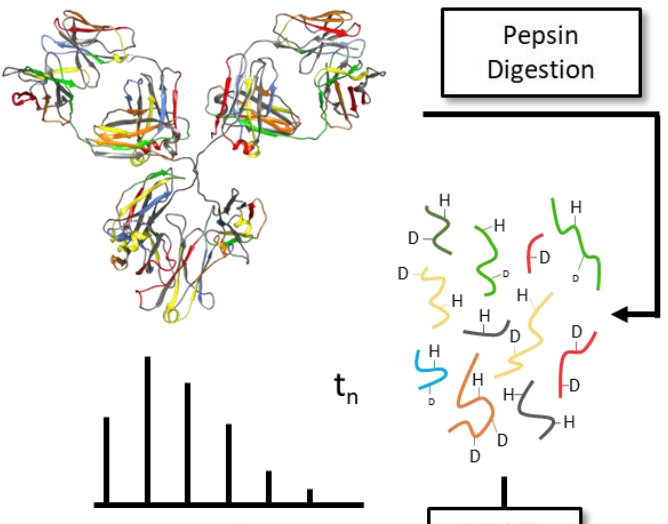

H

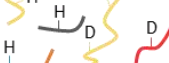

西

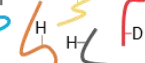

$\sim_{D}>^{-H}$

LC-MS

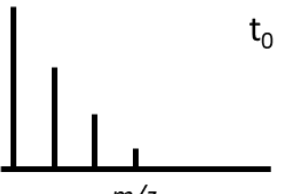

$t_{0}$

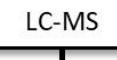

Figure 1: Experimental workflow summarising the methods utilized in this paper in order to assess the conformational spread and populations (d), the conformational stability (e) and the localised dynamics and allosteric effects (f) on Herceptin ${ }^{\circledR}$ between lots and as a function of glycosylation. Intact IgG1s (a) were digested with endoS2 (b) or PNGase $F(c)$ to truncate or remove the $\mathrm{N}$-linked glycosylation, respectively. The three $\mathrm{mAb}$ forms were analysed using ion mobility mass spectrometry and hydrogen/deuterium exchange mass spectrometry. IM-MS investigated the conformational spread (d) of each mAb form in the gas phase and identified different conformational families. Ions of the same mass-to-charge can be separated based upon their size and shape; compact ions will traverse the mobility cell faster than more extended ions. The arrival time distribution is converted into a collision cross section distribution ${ }^{28}$ allowing a comparison of the conformational space occupied by each sample. Collisional activation of the mAbs followed by ion mobility (e) measured the stability profiles of each $m A b$ form and defined the conformational transitions $\left(T_{1}, T_{2}, T_{3}\right)$ across the collision voltage range. HDX-MS measures the solvent accessibility of the $m A b$ backbone (f) and therefore provides insight into the structural dynamics of the protein. The samples are incubated with deuterated buffer for different time points ( $t_{0}$ is the reference and therefore is not deuterated) before the exchange reaction is quenched and the mAb is digested using pepsin. Mass spectrometry measurements calculate the mass shift (level of deuterium uptake) with respect to time providing information regarding the dynamic properties of specific regions on the $m A b$ structure. 


\section{Materials and Methods}

Materials. Herceptin ${ }^{\circledR}$ antibody lots were from Roche-Genentech. NIST monoclonal antibody reference material 8671 was purchased from NIST and the IgG1 Fc-hinge fragment was supplied from a private source. EndoS2 (GlycINATOR ${ }^{\circledR}$ and immobilised GlycINATOR ${ }^{\circledR}$ ) were purchased from Genovis AB. PNGase $F$ and ethylenediaminetetraacetic acid (EDTA) were purchased from Promega. Ammonium acetate, $\mathrm{K}_{2} \mathrm{HPO}_{4}$ and $\mathrm{KH}_{2} \mathrm{PO}_{4}$ were purchased from Fisher Scientific. lodoacetamide, guanidine hydrochloride, trifluoroacetic acid (TFA), and tris (2-carboxyethyl) phosphine hydrochloride (TCEP) were purchased from Sigma-Aldrich. Lysyl Endopeptidase ${ }^{\circledR}$ was purchased from Wako Chemicals and Tris buffer was purchased from Fluka. Purified water was produced in-house using a Milli-Q Advantage A10 system. LC-MS grade acetonitrile and formic acid were purchased from VWR Chemicals and Fluka Analytical, respectively. All samples were desalted using micro Bio-Spin 6 columns (Bio-Rad). To reduce aggregation (<2\%), nESI IM-MS mAb samples were prepared at $1 \mathrm{mg} / \mathrm{ml}(\sim 5 \mu \mathrm{m})$ in $100 \mathrm{mM}$ ammonium acetate, $\mathrm{pH} 6.80$. For HDX-MS mAb samples were prepared at $3 \mathrm{mg} / \mathrm{ml}$ ( $10 \mathrm{mM}$ phosphate, $\mathrm{pH} 7.00$ ), diluted $\sim 30$-fold during the incubation/quench stages resulting in a $<10 \mu \mathrm{g}$ injection size. Previously reported LC-MS analysis of IdeS digested Herceptin ${ }^{\circledR}$ lots calculated Fc/2 and F(ab')2 fragment masses to be within $12 \mathrm{ppm}$ and $41 \mathrm{ppm}$ of the expected masses, respectively [expected masses $25317 \mathrm{Da}$ (average) and $97628 \mathrm{Da}$, respectively]. ${ }^{18}$

Enzymatic Digestions to Selectively Cleave Glycans. mAb samples (50 mM ammonium bicarbonate, $\mathrm{pH} 7.8$ were incubated with PNGase $\mathrm{F}$ at $37^{\circ} \mathrm{C}$ for $20 \mathrm{~h}$, at optimised IgG:enzyme ratios. For endoS2 treated $\mathrm{mAb}$ samples, $1 \mathrm{mg} / \mathrm{ml}$ IgG was incubated at $37^{\circ} \mathrm{C}$ for $30 \mathrm{~min}$ with endoS2 for nESI-IM-MS analyses (Figure S1 in Supplementary Information). For HDX-MS experiments, endoS2 digestion was performed using Immobilised GlycINATOR ${ }^{\circledR}$ (endoS2) cartridges to prevent His-tag interaction with the pepsin column. Prior to nESI-IM-MS or HDX-MS analyses the samples were buffer exchanged into $100 \mathrm{mM}$ ammonium acetate or $10 \mathrm{mM}$ phosphate buffers, respectively. MS spectra to confirm the complete enzymatic digestion of Herceptin ${ }^{\circledR}$, the NIST mAb and the IgG1 Fc-hinge fragment samples are provided in Figures S2, S3 and S4 in the Supplementary Information, respectively.

IM-MS Analysis. IM-MS experiments were acquired using a modified Waters Synapt G2 with a 25.05 cm RF-confining linear drift cell filled with 2 Torr helium, $298 \mathrm{~K}$. A multi-field approach (DV range 213-114 V, 20 V increments) was applied using WREnS and in-house software (ORIGAMI). ${ }^{29}$ For nESI, in-house pulled borosilicate capillary tips (Sutter P-1000 micropipette puller system; capillary ID: 0.9 $\mathrm{mm}, \mathrm{OD}: 1.2 \mathrm{~mm}$ ) were used with platinum wire inserted; capillary voltage $1.2-1.4 \mathrm{kV}$, cone $100 \mathrm{~V}$ and source temperature $40{ }^{\circ} \mathrm{C}$. The arrival time distributions (ATDs) were converted to collision cross section distributions (CCSDs) for the most intense charge states, allowing visualisation of the 
conformational spread adopted by the samples in the gas-phase. Summation of these CCSD plots, (i.e. removing the effects of charge), generates global CCSD plots which provide an overview of the conformational diversity of the sample. The global CCSDs for the NIST mAb were fitted with the minimum number of Gaussians required for the cumulative fit based upon diffusion limitations. Each Gaussian was defined and fixed by the FWHM and apex position before being transposed onto the global CCSDs of the Herceptin ${ }^{\circledast}$ lots.

HDX-MS Analysis. The HDX-MS setup comprised of a Waters nano-Acquity UPLC system with ESI MS detection coupled to a LEAP Technologies dual-armed robot for sample preparation, incubation and inlet injection. A Waters Synapt G2Si mass spectrometer was operated in positive ion/resolution mode, with data acquired over $\mathrm{m} / \mathrm{z}$ range $290-2500$. The LC gradient was supplied at $40 \mu \mathrm{l} / \mathrm{min}$ flow and peptides were eluted over 16 mins with $85 \%$ organic (mobile phases: A, water $+0.1 \%$ formic acid; and $B$, acetonitrile $+0.1 \%$ formic acid). Samples were incubated with deuterated labelling buffer (10 $\mathrm{mM}$ Phosphate in $\mathrm{D}_{2} \mathrm{O}, \mathrm{pD} 7.00$ ) for time points ranging from $0 \mathrm{~min}-8 \mathrm{hrs}$, before being quenched and reduced by quench buffer (0.5 M TCEP, 4 M Guanidine Hydrochloride, $100 \mathrm{mM}$ phosphate, pH 2.30) at $1{ }^{\circ} \mathrm{C}$ for $30 \mathrm{~s}$. On-line pepsin digestion (Waters Enzymate BEH Pepsin $2.1 \times 30 \mathrm{~mm}$ ) at $20^{\circ} \mathrm{C}$ for 3 mins preceded the analytical column held at $0{ }^{\circ} \mathrm{C}$ (Waters Acquity UPLC BEH C18 $1.7 \mu \mathrm{m}, 1.0 \times 10$ $\mathrm{mm}$ ) for chromatographic peptide separation. Data was acquired using Waters MassLynx software v4.1, with the LEAP robot controlled by HDx Director 1.0.3.9. Data processing and analysis were carried out with Waters ProteinLynx Global Server 3.0.1 and Waters DynamX 3.0 software, respectively. For global HDX-MS analysis the pepsin column was replaced with a union, TCEP and Guanidine Hydrochloride were removed from the quench buffer and ToF-MS mode was used for data acquisition. All other method parameters remained unchanged.

Collision Activated IM-MS Analysis. Experiments were performed on a Waters Synapt G2S using nESI and trap-activated ion mobility; capillary voltage $1.2-1.3 \mathrm{kV}$, cone $100 \mathrm{~V}$ and source temperature 40 ${ }^{\circ} \mathrm{C}$. The trap region was pressurised at $\sim 3.22 \mathrm{e}^{-2}$ mbar argon gas and for mobility separation the travelling-wave cell was operated at $\sim 2.85 \mathrm{mbar}$ ( $35 \mathrm{~V}$ wave height, $600 \mathrm{~m} / \mathrm{s}$ wave velocity). The $24^{+}$ charge state (most intense) was mass selected using the quadrupole prior to the trap region to ensure that recorded IM data was a result of ion activation and not charge stripping. ORIGAMI ${ }^{29}$, was used to automatically acquire data for collision energies $4-200 \mathrm{~V}$ in $2 \mathrm{~V}$ increments as well as for data processing.

Disulphide Bond Mapping. $100 \mu \mathrm{g}$ of mAb was incubated for 20 minutes at $57^{\circ} \mathrm{C}$ in denaturing buffer (6M guanidine hydrochloride, $50 \mathrm{mM}$ Tris and $5 \mathrm{mM}$ EDTA, $\mathrm{pH}$ 8). Each sample was prepared in duplicate; to one replicate $2.5 \mu \mathrm{L}$ iodoacetamide ( $300 \mathrm{mM}$ in $200 \mathrm{mM}$ sodium phosphate, $\mathrm{pH}$ 6.5) was 
added and to the other $2.5 \mu \mathrm{L}$ of water was added. A further $100 \mu \mathrm{L}$ of water was added to each sample to dilute the guanidine hydrochloride. Lys-C was added in a $1: 100$ ratio and incubated at $37^{\circ} \mathrm{C}$ overnight. TFA was added to a final concentration of $1 \%$. Samples were analysed via LC-MS using a Waters Acquity UPLC coupled to a Waters Vion mass spectrometer operated in positive ion/resolution mode. The chromatographic separation was achieved using a Waters Acquity BEH C18 Peptide column $(2.1 \times 100 \mathrm{~mm}, 1.7 \mu \mathrm{m})$ and an LC gradient supplied at $0.25 \mathrm{~mL} / \mathrm{min}$, over $90 \mathrm{mins}$ up to $90 \%$ organic (mobile phases; water, acetonitrile and $1 \%$ TFA). Waters UNIFI ${ }^{\mathrm{TM}} 1.8$ was used to generate a list of possible disulphide bond linkages.

Circular Dichroism. Far UV-CD analysis was performed using a Chirascan ${ }^{\mathrm{TM}} \mathrm{CD}$ spectrometer. Data was recorded over a wavelength range of $190-260 \mathrm{~nm}$ with a $0.5 \mathrm{~nm}$ step size. $1 \mathrm{mg} / \mathrm{ml}$ samples in 100 $\mathrm{mM}$ ammonium acetate buffer (concentration measured precisely using a Thermo NanoDrop 2000) were added to a $0.1 \mathrm{~mm}$ cuvette and analysed at $25^{\circ} \mathrm{C}$ for 12 minutes (time per point $=5 \mathrm{sec}$ ). The data was converted from millidegrees (mdeg) to molar ellipticity $([\Theta])$ to correct for deviations in concentration between samples.

Interactive Figures. A number of main text and SI figures presented in this article were recreated in an interactive format to enable in-depth interrogation of the presented results. These are deposited online at https://github.com/BarranLab/Upton mAbs 2018 and are available free of charge. The interactive figures were created using ORIGAMI ${ }^{29}$ and require usage of a modern internet browser and access to the internet.

\section{Results and Discussion}

Demonstrating Conformational Variability between Lots and as a Function of Glycosylation with Native Ion Mobility Mass Spectrometry

Here we compared the distribution of conformers found for each Herceptin ${ }^{\circledast} \mathrm{mAb}$ lot, and whilst broadly similar, each is distinctive (Figure 2a). Drift time CCSD $\left({ }^{\mathrm{DT}} \mathrm{CCSD}_{\mathrm{He}}\right)$ plots show the highest similarity between Herceptin ${ }^{\circledR}$ lots $A$ and $B$, whereas lot $C$ possesses a comparably broader conformational profile. This analysis allows us to measure conformational lot-to-lot variability in the API. Intact mAbs are inherently heterogeneous consisting various glycoforms in many structural orientations, to mitigate this enzymatic treament can be used to dissect these glycan moeities resulting in a more homogenous molecule. Treating glycans with either PNGase $\mathrm{F}$ to deglycosylate or endoS2 to truncate (i.e. GICNAc or [GIcNAc + Fucose] remaining) renders the CCS distributions more similar, yet notable conformational differences remain (Figure 2Error! Reference source not found.bc). The NIST mAb was analysed in the same way and presents a conformational spread most similar to 
those of Herceptin ${ }^{\circledast}$ lot $C$, indicating that the glycosylation in this lot is most variable and that this causes observable conformational differences.

Native mass spectrometry approaches have shown that proteins can retain a number of different conformations even in the absence of bulk solution, which can be related to solvated data. ${ }^{30,31}$ For the NIST mAb eight conformational familes were fitted across the intact, endoS2 treated and deglycosylated samples (Figure $\mathbf{2 d - f}$ ). The most compact conformer in the intact sample (I) contracts $\sim 3.5 \%$ (conformers III and VI) following glycan modification whilst introducing more extended conformers (V and VIII). Transposing these defined, Gaussian-fitted conformations onto the Herceptin ${ }^{\circledR}$ lots allows visualisation of the conformational space occupied, relative to a standard (Figure S5 in Supplementary Information). The distributions present in the NIST mAb align well with the global CCSDs measured for Herceptin ${ }^{\circledR}$ lots A and B, whereas Herceptin ${ }^{\circledR}$ lot C presents a notably more extended profile with the addition of a ninth conformational family occupied in the endoS2 treated sample (Figure S5 in Supplementary Information). Tracking the occupany of each conformation across all endoS2 treated and deglycosylated samples allows the lots to be compared (Figure $\mathbf{2 g}$ and $\mathbf{2 h}$ ). In Figure $\mathbf{2 g}$ the additional fourth conformer (orange star) for endoS2 treated Herceptin ${ }^{\circledR}$ lot $C$ indicates destabilisation $c f$. the other samples. The mAb structures in Figures $\mathbf{2 d - f}$ illustrate the conformational collapse which is observed following glycan truncation or removal. These have been adapted from previously reported molecular dynamic (MD) simulations of an IgG1 confirmed that despite a conformational collapse, secondary structure is largely retained. ${ }^{22}$ 


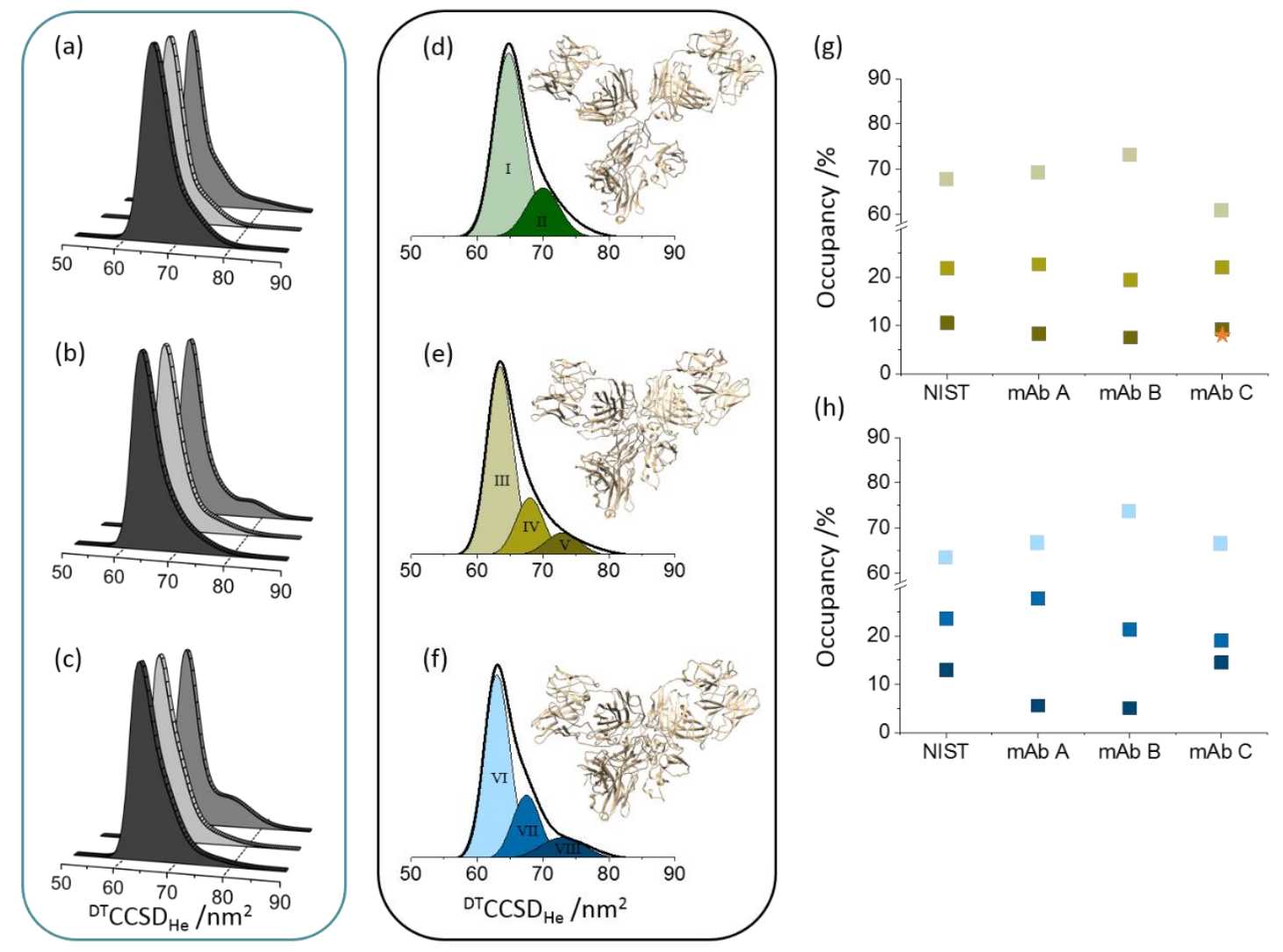

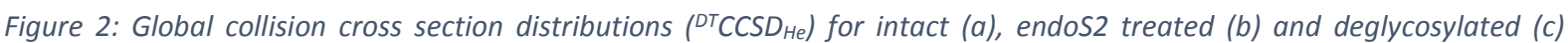
Herceptin ${ }^{\circledR}$; lot A (dark grey), lot B (light grey) and lot C (grey). Global CCSDs for intact (d), endoS2 treated (e) and deglycosylated (f) NIST mAb standard with fitted conformers. The darker the Gaussian for each sample, the more extended the conformer. The conformers present in each sample preparation have slightly different centres and FWHM values, hence different colours for each sample preparation. CCSDs for individual charge states for each sample and at each glycosylation level are provided in Figure $\mathbf{S} 6$ in Supplementary Information. Inserted mAb figures are snapshots from MD trajectories previously performed on pdb: $1 / G Y .22$ The image in (d) is from early in the MD simulation and therefore represents the intact $m A b$; later snapshots represent more contracted forms (e and f). Percentage occupancies of each conformer in the endoS2 treated samples $(\mathrm{g})$ and deglycosylated samples $(\mathrm{h})$ are presented with colours corresponding to the fitted Gaussians. For the endoS2 treated Herceptin ${ }^{\circledR}$ lot $\mathrm{C}$ there is an additional fourth extended Gaussian (see Figure 55 in Supplementary Information) which is represented by the orange star.

CCSD plots, both at the local and global level, were also produced for an IgG1 Fc-hinge fragment (Supplementary Information Figure S7) to detail conformational changes in the $\mathrm{C}_{\mathrm{H}} 2$ and $\mathrm{C}_{\mathrm{H}} 3$ domains of IgG1s. The data supports the observations made in the full mAb IM-MS analyses Figure 2 and Figure S5 where flexibility is enumerated as the width of the CCSDs. Taken together both the Fc-hinge fragment and the full IgG1s change in structure as a function of glycosylation in the following order intact $<$ deglycosylated $<$ endoS2 treated.

Intrinsic Flexibility of mAbs is Revealed by Collisional Activation Followed by Ion Mobility Mass Spectrometry

Collisional activation coupled with IM-MS analysis can be used to probe changes in structure and how these couple to fragmentation for any given molecular ion. ${ }^{29,31-34}$ This method has been previously applied to compare the behaviour of one mAb lot as a function of glycosylation both in an intact lgG1 
and an IgG1 Fc-fragment (IdeS digested below the hinge). ${ }^{35}$ Comparing each of the samples following sequential enzymatic truncation of the glycans (six glycosylated states in total) against the fully glycosylated mAb, Tian et al. demonstrated that the collisional activation energy required to trigger the conformational transitions correlated to the size of the glycan; where increased glycan size provided increased stabilization towards conformational changes.

Here we use a similar approach to compare across Herceptin ${ }^{\circledast}$ lots as well as three levels of glycosylation. To quantify differences, ORIGAMI ${ }^{29}$ subtraction plots were generated along with the root-mean-square-deviation (RMSD, Figures 3d and 3e). Differences in RMSD across the Herceptin ${ }^{\circledast}$ lots with the same extent of glycosylation demonstrate lot-to-lot variations (Table S1 in the Supplementary Information). The unfolding profiles for lots A and B are highly similar evidenced by the low RMSD values. When compared with lot $C$ however, the unfolding transitions occur at lower collision voltages indicating reduced stability towards collisional activation compared with lots $\mathrm{A}$ and B. The RMSD values and heat maps for the Herceptin ${ }^{\circledR}$ lots and the NIST mAb standard with varying levels of glycosylation, are summarised in Figure 3e and Supplementary Information Figures S8-S11. As the extent of glycosylation is reduced the RMSD value, when compared with the fully glycosylated form, increases and the collision voltage required to induce the distinct conformational transitions is reduced. There is an inherent stability induced by glycosylation and truncation of the glycans using endoS2 preserves more stability compared to complete glycan removal.

In order to provide more detail on the observed conformational changes we can compare the behaviour of the Herceptin ${ }^{\circledR} \mathrm{Fc}$-hinge fragment as a function of glycosylation and contrast it with the findings of Tian et al. who examined Fc/2 (Figure 3 and Table S2 in Supplementary Information). When fully deglycosylated the Fc-hinge fragment more readily transitions (as shown by the comparison of the inflexions to the dotted lines $T_{1}, T_{2}$ and $T_{3}$ ), at collision voltages significantly lower $(27-39 \%)$ than those required at the intact level. Although the stability trends are the same across the mAb samples and the Fc-hinge fragment, the increase in RMSD for the fragment sample means there is a clearer distinction between the different levels of glycosylation (Figure 3e). We attribute these differences in RMSD magnitude to the proportion of glycosylation relative to the protein; for the intact mAb, glycosylation accounts for $\sim 2 \%$ mass whereas this increases to $\sim 6 \%$ for the IgG1 Fchinge fragment. Decreasing the protein size, increases the potential sensitivity of the activated IM-MS experiment and therefore enhances the relative impact of altering glycan structure. The effect of glycosylation upon Fc conformation has been well documented ${ }^{36,37}$ and with increased experimental sensitivity, structural and stabilisation effects across samples will be more apparent when compared with the intact $m A b$. 

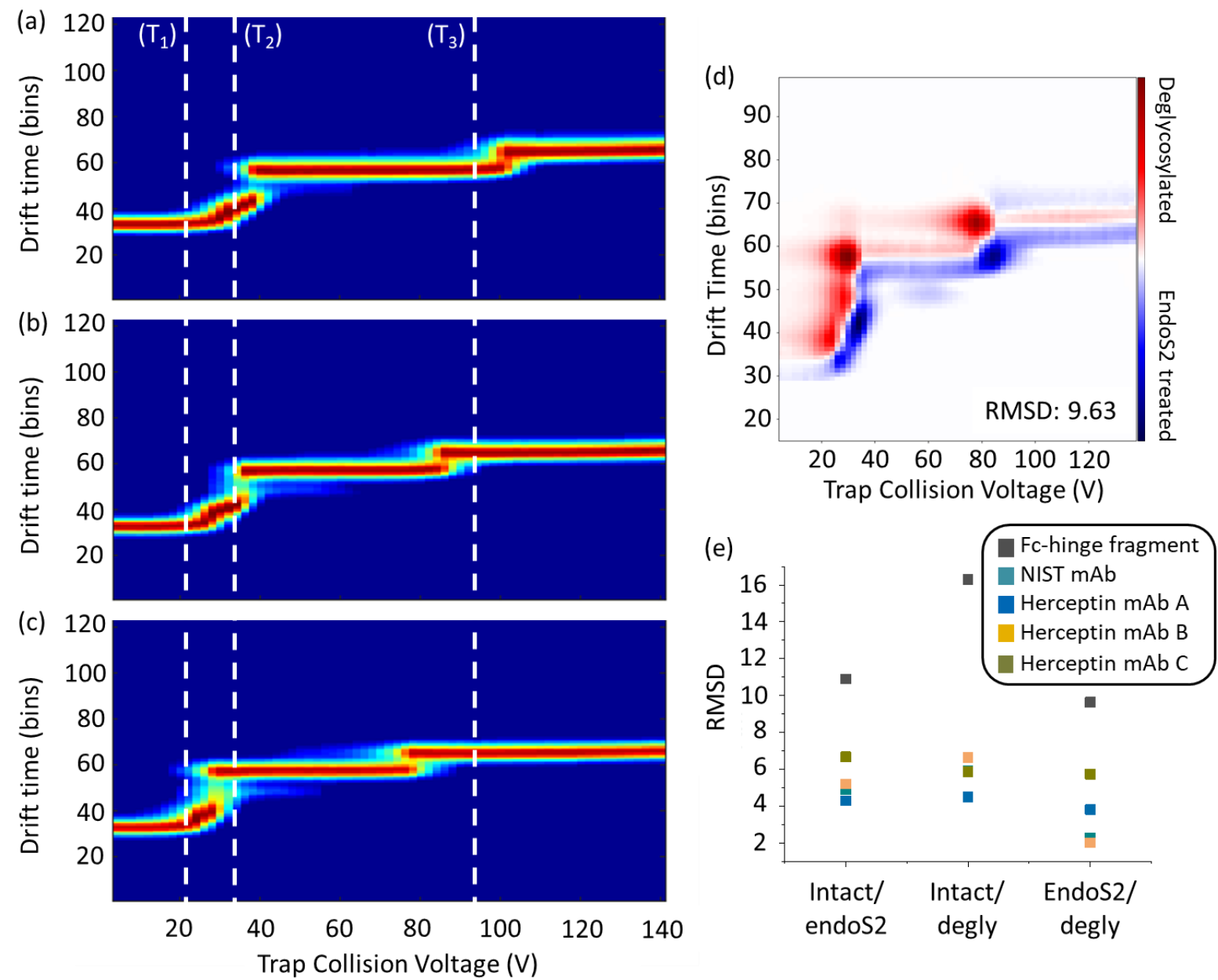

Figure 3: Ion mobility mass spectrometry heat maps as a function of collisional activation energy of an IgG1 Fc-hinge fragment with glycans (a), truncated (endosS2 treated) glycans (b) and no glycans (c). White dashed lines represent the start of a conformational transition $\left(T_{1}, T_{2}\right.$ and $T_{3}$ ) relative to the intact sample; associated quantitative collision energy values are reported in Table S2 in the Supplementary Information. Data shown for the mass selected $14^{+}$charge state with collision voltage range 4-140 V. Example activated IM-MS difference plot for the comparison of endoS2 treated and deglycosylated Fc-hinge fragments (d) along with calculated RMSD values for all samples at all levels of comparison (e). Heat maps for the IgG1 mAbs are provided in Figures S8-S11 in the Supplementary Information.

\section{What is the Difference in Solvent Accessibility of the Lots in Solution and can we Map it to the}

\section{Tertiary Fold?}

HDX-MS monitors the isotopic exchange of hydrogen for deuterium along the protein backbone as a mass shift with respect to analysis time ${ }^{38}$ By mapping regions of solvent accessibility onto the protein structure, HDX-MS offers information regarding the tertiary fold, flexibility and average conformation of the protein in solution over an experimental time scale that can range from seconds to days. ${ }^{39} \mathrm{We}$ used HDX-MS experiments to monitor the effects of varying levels of glycosylation upon the mAb dynamics of Herceptin ${ }^{\circledR}$ lots A and C, the Fc-hinge fragment and the Waters mAb as a standard. (For these experiments the NIST mAb was not used as the HDX-MS sequence coverage achieved was $\sim 58$ $\%$ compared with $70-80 \%$ achieved with the other samples. The NIST mAb can be a difficult protein to work with and has been stated to provide variable HDX-MS sequence coverages (Jeffrey Hudgens, 
Personal Communication, 26 March, 2018)). Each sample was analysed in the fully glycosylated form (intact), after endoS2 digestion and following deglycosylation. Observations made across all samples were that for most peptides ( $81 \%$ ) deuterium uptake increased according to the trend, intact < deglycosylated < endoS2 treated (see Supplementary Information Figures S12 and S13 for examples). For the Herceptin ${ }^{\circledR}$ mAb lots the uptake difference between the three sample preparations was generally greater than for the Waters mAb standard; with reduced comparability between the endoS2 treated $\mathrm{mAb}$ peptides and the deglycosylated mAb peptides from the mAb standard (Supplementary Information Figures S12 and S13). The complete peptide uptake difference plots for the Waters mAb standard (Supplementary Information Figure S14) confirm the increased deuterium uptake in the absence of part or full glycan moieties, with minimal difference between the two.

A comparison of intact vs. endoS2 treated Herceptin ${ }^{\circledR}$ (Figure 4), was made by subtracting the endoS2 uptake data from the intact uptake data for each identified peptide along the heavy chain for both Herceptin ${ }^{\circledast}$ lots $A$ and $C$ independently. Subtracting within individual samples enables direct comparison between samples as the results are independent of differences in intact uptake i.e. provided a direct comparison of the effects of endoS2 treatment upon the intrinsic stability of the Herceptin ${ }^{\circledR}$ lots. In both Herceptin ${ }^{\circledR}$ lots there was a significant increase in uptake across the heavy chain following endoS2 digestion, implying significant structural alterations leading to the availability of more solvent accessible hydrogen atoms. A plausible explanation for this is that the glycan 'stubs' (single GlcNAc or [GlcNAc + Fucose]) interact with one another to 'hold-open' the Fc-domain, increasing the solvent accessibility without the steric hindrance of the intact glycan. 


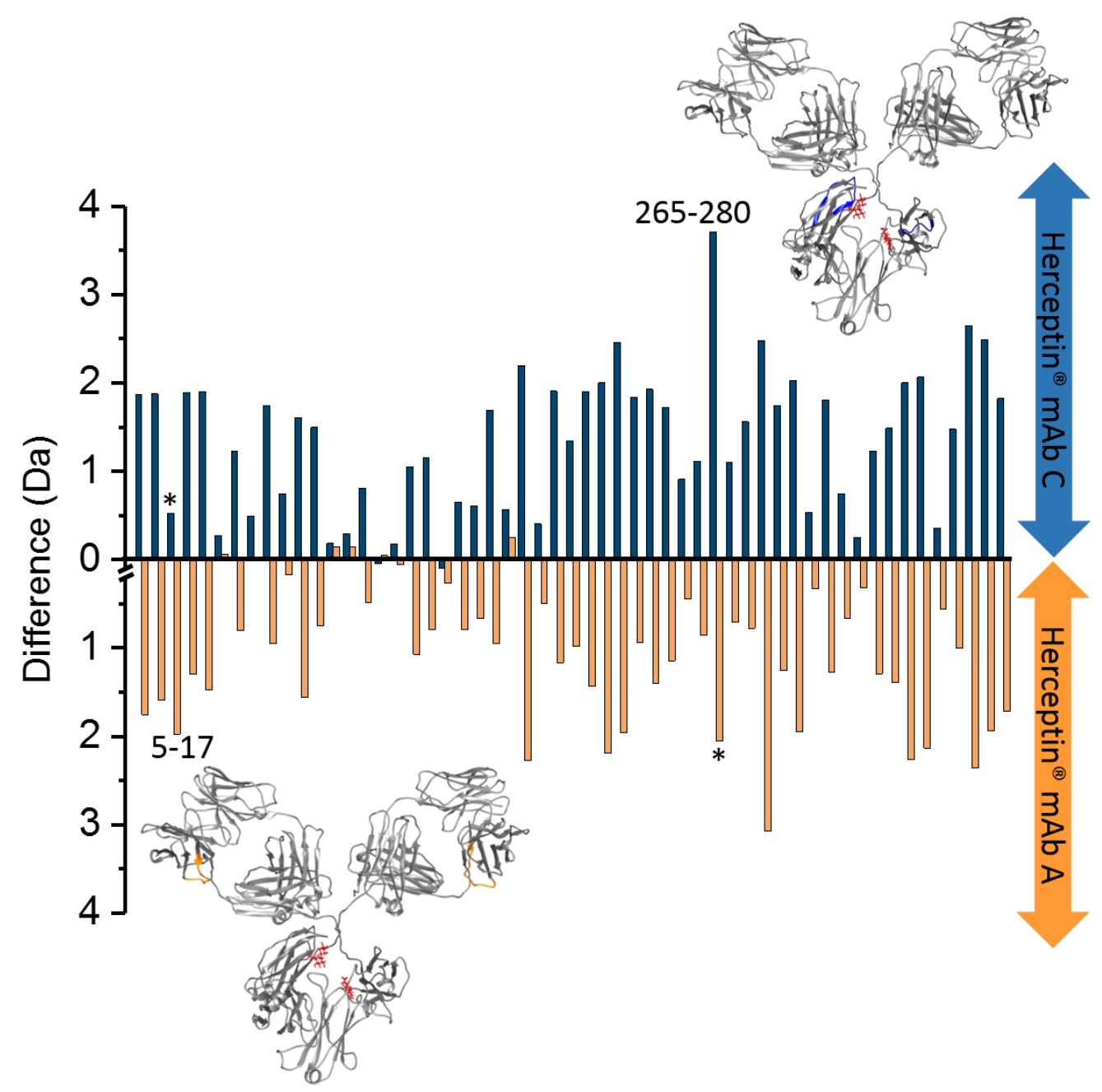

Figure 4: Deuterium uptake difference plots for heavy chain (HC) intact vs. endoS2 treated Herceptin ${ }^{\circledR}$; endoS2 - intact. Each bar represents a different peptide (HC sequence coverage $=70.2 \%, 55$ peptides). Blue bars represent the deuterium uptake differences for Herceptin ${ }^{\circledR}$ lot C; orange bars represent deuterium uptake differences for Herceptin ${ }^{\circledR}$ lot A. Labelled peptides 5-17 (VESGGGLVQPGGS) and 265-280 (VVVDVSHEDPEVKFNW) are those with significant uptake differences between the two Herceptin ${ }^{\circledR}$ lots i.e. a difference of $>1$ Da (see Supplementary Information Figure S15 for the Herceptin ${ }^{\circledR}$ lot C - lot A subtraction plot); * corresponds to the equivalent peptide in the other sample. The peptide locations for the two peptides with uptake differences $>1 \mathrm{Da}$ are highlighted on the mAb structures in blue and orange for lot $C$ and lot $A$, respectively. A representation of endoS2 treated glycans are shown in red. An interactive version of this Figure is available at the following link; $\underline{\text { http://bit.ly/2OjV3R6. }}$

By subtracting the two sets of data presented in Figure 4 from one another, differences between Herceptin ${ }^{\circledR}$ lot $C$ and lot A were seen (Supplementary Information Figure S15), with the majority of peptides showing an increased deuterium uptake in Herceptin ${ }^{\circledR}$ lot $\mathrm{C}$ compared with lot $A$. The peptides with most significant differences (peptides 5-17 and 265-280) are highlighted on the mAb structures in Figure 4 (and in Supplementary Information Figure S15) with the location of all peptides shown in Figure 5. Peptides close to the site of glycosylation (281-296 and 310-321 for example) were understandably affected by the truncation of the glycans, and interestingly so were a number of peptides in the Fab region; implying that alterations to the Fc region conformation also causes conformational changes in the Fab region. Intact vs. deglycosylated heavy chain data and endoS2 vs. deglycosylated heavy chain data are shown in Supplementary Information Figures S16 and S17, 
respectively. There are clear similarities between Supplementary Information Figures S15 and S16, confirming that both glycan truncation and removal increases deuterium uptake to a greater extent in Herceptin ${ }^{\circledR}$ lot $\mathrm{C}$ compared with lot $\mathrm{A}$. The quantitative differences between the two glycosylation levels are presented in Supplementary Information Figure S17; no significant differences are observed (at the $>1$ Da level), however nearly all peptides in Herceptin ${ }^{\circledR}$ lot C show increased uptake compared with lot $A$.

These same uptake trends were observed in the Herceptin ${ }^{\circledast}$ light chain data and for the Fc-hinge fragment (Supplementary Information Figures S18 and S19, respectively). The Fc-hinge fragment, yields similar HDX data from both endoS2 treated and deglycosylated samples, with four of the five peptides/regions with significant uptake differences (> $1 \mathrm{Da}$ ) in both treated samples compared with the intact sample. For the endoS2 treated Fc-hinge fragment an additional peptide (166-183) was significant (Supplementary Information Figure S19). All HDX-MS data was analysed in duplicate with the same uptake trends observed across all repeats and samples. 


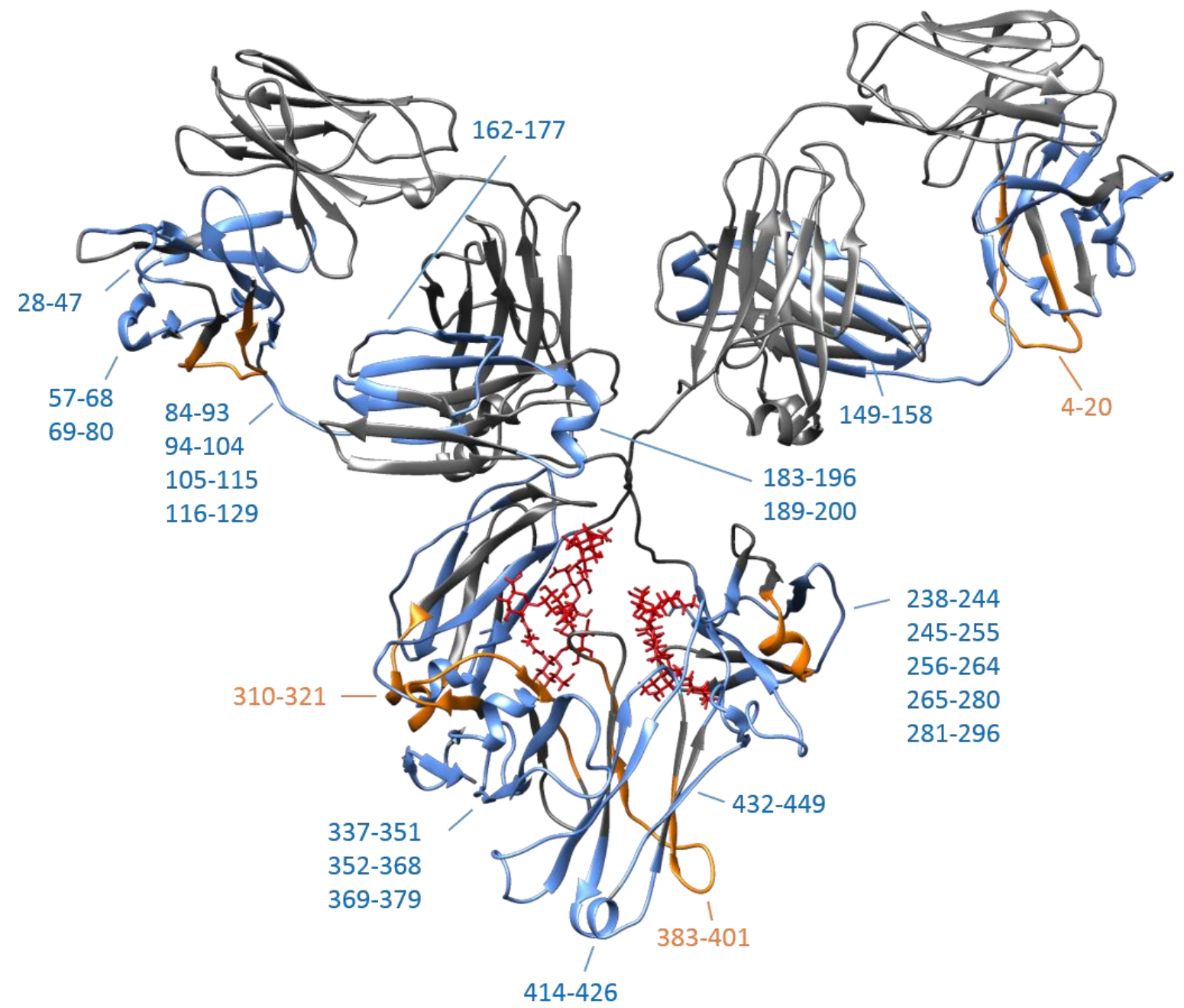

Figure 5: IgG1 schematic ( $p d b: 1 / G Y$ ) depicting the location of all heavy chain peptides shown in Supplementary Information Figure $\mathbf{S 1 5}$ for intact vs. endoS2 treated Herceptin ${ }^{\circledR}$ lots. Peptides/ consecutive peptide regions where the average greatest uptake is in lot $C$ are shown in blue and are shown in orange for peptides from lot A. Original intact glycan positions shown in red. Heavy chain peptide coverage maps are provided in Supplementary Information Figures S20 and S21.

\section{Orthogonal techniques to assess lot-to-lot variability}

Disulphide bond mapping was performed on each of the Herceptin lots to confirm that the conformational differences discussed above were not as a result of non-canonical cysteine pairings. A comparison of the proteolytic digests from the alkylated and non-alkylated samples identified the likely disulphide bonds within the starting material (Table S3 in the Supplementary Information). The lys- $C$ digest revealed no differences in the protein sequences, no deviations from the canonical disulphide bridge pattern expected and no PTM variations across the lots.

Circular Dichroism was used to assess the secondary structure of the Herceptin lots and to allow comparison with the NIST mAb. Following conversion to molar ellipticity units, unlike the MS approaches describe above this method did not reveal any observable differences between the lots (Figure S22 in the Supplementary Information). 


\section{Conclusions}

We have shown that native mass spectrometry can be usefully employed to distinguish between lots of an active biopharmaceutical and demonstrated a method by which data can be compared with that obtained from a standard. The results present significant conformational similarities between two of the Herceptin ${ }^{\circledR}$ lots however, lot $\mathrm{C}$ exists in a wider range of conformational states demonstrated by both HDX-MS and IM-MS. We have also mapped how variations in $\mathrm{N}$-linked Fc-domain glycosylation affect the intrinsic conformations of IgG1 mAbs. HDX-MS shows how deglycosylation causes more solvent exposure in both the Fc and Fab domains, and in vacuo analysis indicates a concomitant conformational contraction/collapse and higher flexibility upon collisional activation of the mAb. Similar observations are made for endoS2 treated samples, compared with the intact glycosylated samples, although the deuterium uptake for the majority of peptides was greater than for the equivalent deglycosylated samples. Taken together these findings indicate that the truncated glycan 'stubs' (single GlcNAc or [GIcNAc + Fucose]) interact within the Fc-domain, creating a solvent accessible pocket with reduced steric hindrance. The IM-MS data supports this also with the widest conformational spread being observed for the endoS2 treated Fc-hinge fragment and with the retention of some stability demonstrated by the activated IM-MS heat maps. As for the comparisons across different lots of Herceptin ${ }^{\circledR}$, the conformational trends (endos2 treated > deglycosylated > glycosylated) across the techniques are the same. However the magnitudes differ particularly for Herceptin ${ }^{\circledR}$ lot $\mathrm{C}$. With all three lots having approval for clinical use from the relevant authorities, these findings indicate the range of measurable conformational properties that may fulfil acceptance criteria. With this in mind it is no trivial matter defining all critical quality attributes for complex biopharmaceuticals, and full characterisation of originator lots should be coupled with clinical consequences for example the level of afucosylation, before embarking upon biosimilar classification. In this work we have considered three lots of Herceptin ${ }^{\circledR}$ and three levels of glycosylation (intact, truncated and fully deglycosylated). Going forward it would be desirable to extend these techniques to explore a wider range of glycan occupancies, coupled with sequential enzymatic cleavages. We recommend that native MS methods could have a very useful role for initial characterisation as they are rapid and lend well to comparability studies. HDX-MS provides more detail, but at the cost of significant machine and analysis time and for some systems the reproducibility is poor. Analysis of biosimilar candidates in comparison with a wider range of originators would also help cement these techniques and the information provided within a robust, regulatory biosimilar characterisation workflow. 


\section{Acknowledgements}

This work has been funded by a BBSRC Industrial Case Studentship awarded to RU in collaboration with Covance Laboratories. The authors would like to thank Dr. Eddie McKenzie from the Manchester Protein Structure Facility for discussions had and Dr. Derren Heyes from the Manchester Centre for Biophysics and Catalysis for help with the CD experiments. Thank you to Mark Beaton from Waters for establishing our HDX-MS system.

\section{Supporting Information}

Supporting results (Figures S1-S22 and Tables S1-S3) as mentioned in the text are available free of charge.

\section{References}

1. Ecker, D. M., Jones, S. D. \& Levine, H. L. The therapeutic monoclonal antibody market. MAbs 7, 9-14 (2015).

2. Roger, S. D. Biosimilars: how similar or dissimilar are they? Nephrology (Carlton). 11, 341-6 (2006).

3. Rosati, S., Yang, Y., Barendregt, A. \& Heck, A. J. R. Detailed mass analysis of structural heterogeneity in monoclonal antibodies using native mass spectrometry. Nat. Protoc. 9, 96776 (2014).

4. Wang, X., An, Z., Luo, W., Xia, N. \& Zhao, Q. Molecular and functional analysis of monoclonal antibodies in support of biologics development. Protein Cell 9, 74-85 (2018).

5. European Medinces Agency: Guideline on immunogenicity assessment of monoclonal antibodies intended for in vivo clinical use. (2012).

6. European Medicines Agency: Guideline on similar biological medicinal products containing monoclonal antibodies - non-clinical and clinical issues. (2012).

7. US Department of Health and Human Services Food and Drug Administration (CBER), Center for Drug Evaluation and Research (CDER) Evaluation and Research: Scientific Considerations in Demonstrating Biosimilarity to a Reference Product. (2015).

8. US Food and Drug Administration. (2015). Available at: http://www.fda.gov/downloads/DrugsGuidanceComplianceRegulatorylnformation/Guidance s/UCM291128.pdf.

9. WHO Expert Committee on Biological Standardization, sixty-seventh report. Geneva: World Health Organisation. WHO Technical Report Series (2017).

10. Wang, J. \& Chow, S. C. On the regulatory approval pathway of biosimilar products. Pharmaceuticals 5, 353-368 (2012).

11. Krapp, S., Mimura, Y., Jefferis, R., Huber, R. \& Sondermann, P. Structural analysis of human IgG-Fc glycoforms reveals a correlation between glycosylation and structural integrity. J. Mol. Biol. 325, 979-989 (2003).

12. Reusch, D. \& Tejada, M. L. Fc glycans of therapeutic antibodies as critical quality attributes. 
Glycobiology 25, 1325-1334 (2015).

13. Berkowitz, S. a, Engen, J. R., Mazzeo, J. R. \& Jones, G. B. Analytical tools for characterizing biopharmaceuticals and the implications for biosimilars. Nat. Rev. Drug Discov. 11, 527-40 (2012).

14. Beck, A. et al. Cutting-edge mass spectrometry characterization of originator, biosimilar and biobetter antibodies. J. Mass Spectrom. 50, 285-297 (2015).

15. Thompson, N. J., Rosati, S., Rose, R. J. \& Heck, A. J. R. The impact of mass spectrometry on the study of intact antibodies: from post-translational modifications to structural analysis. Chem. Commun. 49, 538-48 (2013).

16. Tian, Y. \& Ruotolo, B. T. The growing role of structural mass spectrometry in the discovery and development of therapeutic antibodies. Analyst 143, 2459-2468 (2018).

17. Gilar, M. et al. Characterization of glycoprotein digests with hydrophilic interaction chromatography and mass spectrometry. Anal. Biochem. 417, 80-88 (2011).

18. Upton, R. et al. Orthogonal Assessment of Biotherapeutic Glycosylation: A Case Study Correlating N-Glycan Core Afucosylation of Herceptin with Mechanism of Action. Anal. Chem. 88, 10259-10265 (2016).

19. Damen, C. W. N. et al. Electrospray ionization quadrupole ion-mobility time-of-flight mass spectrometry as a tool to distinguish the lot-to-lot heterogeneity in $\mathrm{N}$-glycosylation profile of the therapeutic monoclonal antibody trastuzumab. J. Am. Soc. Mass Spectrom. 20, 2021-33 (2009).

20. Tian, Y., Han, L., Buckner, A. C. \& Ruotolo, B. T. Collision Induced Unfolding of Intact Antibodies: Rapid Characterization of Disulfide Bonding Patterns, Glycosylation, and Structures. Anal. Chem. acs.analchem.5b03291 (2015). doi:10.1021/acs.analchem.5b03291

21. Jensen, P. F. et al. Investigating the Interaction between the Neonatal Fc Receptor and Monoclonal Antibody Variants by Hydrogen/Deuterium Exchange Mass Spectrometry. Mol. Cell. Proteomics 14, 148-161 (2015).

22. Pacholarz, K. J. et al. Dynamics of intact immunoglobulin $\mathrm{G}$ explored by drift-tube ion-mobility mass spectrometry and molecular modeling. Angew. Chemie - Int. Ed. 53, 7765-7769 (2014).

23. Jones, L. M. et al. Complementary MS methods assist conformational characterization of antibodies with altered S-S bonding networks. J. Am. Soc. Mass Spectrom. 24, 835-845 (2013).

24. Houde, D., Peng, Y., Berkowitz, S. A. \& Engen, J. R. Post-translational Modifications Differentially Affect IgG1 Conformation and Receptor Binding. Mol. Cell. Proteomics 9, 17161728 (2010).

25. Sjögren, J. et al. EndoS and EndoS2 hydrolyze Fc-glycans on therapeutic antibodies with different glycoform selectivity and can be used for rapid quantification of high-mannose glycans. Glycobiology 25, 1053-1063 (2015).

26. NIST Monoclonal Antibody Reference Material 8671. (2018). Available at: https://www.nist.gov/programs-projects/nist-monoclonal-antibody-reference-material-8671. (Accessed: 20th April 2018)

27. Schiel, J. E., Mire-Sluis, A. \& Davis, D. Monoclonal antibody therapeutics: The need for biopharmaceutical reference materials. in State-of-the-Art and Emerging Technologies for Therapeutic Monoclonal Antibody Characterization Volume 1. Monoclonal Antibody 
Therapeutics: Structure, Function, and Regulatory Space (eds. Schiel, J. E., Davis, D. L. \& Borisov, O. V.) 1176, 1-34 (ACS Symposium Series, 2014).

28. Jarrold, M. F. Unfolding, refolding, and hydration of proteins in the gas phase. Acc. Chem. Res. 32, 360-367 (1999).

29. Migas, L. G., France, A. P., Bellina, B. \& Barran, P. E. ORIGAMI: A software suite for activated ion mobility mass spectrometry (aIM-MS) applied to multimeric protein assemblies. Int. J. Mass Spectrom. 427, 20-28 (2018).

30. Shi, H., Pierson, N. A., Valentine, S. J. \& Clemmer, D. E. Conformation types of ubiquitin $[\mathrm{M}+8 \mathrm{H}] 8+$ ions from water:methanol solutions: Evidence for the $\mathrm{N}$ and $\mathrm{A}$ states in aqueous solution. J. Phys. Chem. B 116, 3344-3352 (2012).

31. Beveridge, R. et al. Mass spectrometry locates local and allosteric conformational changes that occur on cofactor binding. Nat. Commun. 7, 1-9 (2016).

32. von Helden, G., Gotts, N. G. \& Bowers, M. T. Experimental evidence for the formation of fullerenes by collisional heating of carbon rings in the gas phase. Nature 363, 60-63 (1993).

33. Dixit, S. M., Polasky, D. A. \& Ruotolo, B. T. Collision induced unfolding of isolated proteins in the gas phase: past, present, and future. Curr. Opin. Chem. Biol. 42, 93-100 (2018).

34. Sivalingam, G. N., Yan, J., Sahota, H. \& Thalassinos, K. Amphitrite: A program for processing travelling wave ion mobility mass spectrometry data. Int. J. Mass Spectrom. 345-347, 54-62 (2013).

35. Tian, Y. \& Ruotolo, B. T. Collision induced unfolding detects subtle differences in intact antibody glycoforms and associated fragments. Int. J. Mass Spectrom. 425, 1-9 (2018).

36. Zheng, K., Bantog, C. \& Bayer, R. The impact of glycosylation on monoclonal antibody conformation and stability. MAbs 3, 568-576 (2011).

37. Hristodorov, D. et al. Generation and comparative characterization of glycosylated and aglycosylated human IgG1 antibodies. Mol. Biotechnol. 53, 326-335 (2013).

38. Konermann, L., Pan, J. \& Liu, Y.-H. Hydrogen exchange mass spectrometry for studying protein structure and dynamics. Chem. Soc. Rev. 40, 1224-1234 (2011).

39. Wei, H. et al. Hydrogen/deuterium exchange mass spectrometry for probing higher order structure of protein therapeutics: Methodology and applications. Drug Discov. Today 19, 95102 (2014). 